\title{
The IRAM-30m line survey of the Horsehead PDR
}

\section{I. $\mathrm{CF}^{+}$as a tracer of $\mathrm{C}^{+}$and as a measure of the fluorine abundance}

\author{
V. Guzmán ${ }^{1}$, J. Pety ${ }^{1,2}$, P. Gratier ${ }^{1}$, J. R. Goicoechea ${ }^{3}$, M. Gerin ${ }^{2}$, E. Roueff ${ }^{4}$, and D. Teyssier ${ }^{5}$
}

1 IRAM, 300 rue de la Piscine, 38406 Saint Martin d'Hères, France e-mail: [guzman; pety; gratier] @iram.fr

2 LERMA - LRA, UMR 8112, Observatoire de Paris and École normale Supérieure, 24 rue Lhomond, 75231 Paris, France e-mail: maryvonne.gerin@lra.ens.fr

3 Centro de Astrobiología, CSIC-INTA, Carretera de Ajalvir, Km 4, Torrejón de Ardoz, 28850 Madrid, Spain e-mail: jr.goicoechea@cab.inta-csic.es

${ }^{4}$ LUTH UMR 8102, CNRS and Observatoire de Paris, Place J. Janssen, 92195 Meudon Cedex, France e-mail: evelyne.roueff@obspm.fr

5 European Space Astronomy Centre, ESA, PO Box 78, 28691 Villanueva de la Caada, Madrid, Spain e-mail: dteyssier@sciops.esa.int

Received 20 April 2012 / Accepted 2 June 2012

\section{ABSTRACT}

\begin{abstract}
$\mathrm{C}^{+}$is a key species in the interstellar medium, but its $158 \mu \mathrm{m}$ fine structure line cannot be observed from ground-based telescopes. Current models of fluorine chemistry predict that $\mathrm{CF}^{+}$is the second-most important fluorine reservoir in regions where $\mathrm{C}^{+}$is abundant. We detected the $J=1-0$ and $J=2-1$ rotational lines of $\mathrm{CF}^{+}$with high signal-to-noise ratio toward the photo-dissociation region and dense core positions in the Horsehead. Using a rotational diagram analysis, we derive a column density of $N\left(\mathrm{CF}^{+}\right)=(1.5-2.0) \times$ $10^{12} \mathrm{~cm}^{-2}$. Because of the simple fluorine chemistry, the $\mathrm{CF}^{+}$column density is proportional to the fluorine abundance. We thus infer the fluorine gas-phase abundance to be $\mathrm{F} / \mathrm{H}=(0.6-1.5) \times 10^{-8}$. Photochemical models indicate that $\mathrm{CF}^{+}$is found in the layers where $\mathrm{C}^{+}$is abundant. The emission arises in the UV-illuminated skin of the nebula, tracing the outermost cloud layers. Indeed, $\mathrm{CF}^{+}$ and $\mathrm{C}^{+}$are the only species observed to date in the Horsehead with a double-peaked line profile caused by kinematics. We therefore propose that $\mathrm{CF}^{+}$, which is detectable from the ground, can be used as a proxy of the $\mathrm{C}^{+}$layers.
\end{abstract}

Key words. astrochemistry - ISM: clouds - ISM: molecules - ISM: individual objects: Horsehead nebula - radio lines: ISM

\section{Introduction}

$\mathrm{C}^{+}$is a key species in the interstellar medium. First, it is an important tracer of the neutral gas where $\mathrm{CO}$ has not yet been able to form (Langer et al. 2010). Second, it is the dominant gas phase reservoir of carbon in the diffuse interstellar medium and its fine structure transition $\left({ }^{2} \mathrm{P}_{3 / 2}-{ }^{2} \mathrm{P}_{1 / 2} 157.8 \mu \mathrm{m}, 1.9 \mathrm{THz}\right)$ is the main cooling mechanism of the diffuse gas. Unfortunately, its rest-frame emission cannot be observed from ground-based telescopes. With Herschel and SOFIA it is now possible to observe this line from space and from the stratosphere, respectively, but with limited spatial resolution ( $12^{\prime \prime}$ and $15^{\prime \prime}$, respectively). It is therefore of great interest to find tracers of $\mathrm{C}^{+}$that can be observed from the ground at much higher spatial resolution, for example with the Atacama Large Millimeter Array (ALMA) or the NOrthern Extended Millimeter Array (NOEMA).

The chemistry of fluorine was studied by Neufeld et al. (2005). Although there are still uncertainties in some reaction rate coefficients (e.g. $\mathrm{CF}^{+}$photodissociation), the current models predict that $\mathrm{CF}^{+}$is present in regions where $\mathrm{C}^{+}$and $\mathrm{HF}$ are abundant, because it is produced by reactions between these species and destroyed mainly by electrons. In these regions $\mathrm{CF}^{+}$is the second-most important fluorine reservoir after HF. The ground rotational transition of $\mathrm{HF}$, which lies at $\mathrm{THz}$ frequencies, was first detected with the Herschel/HIFI in the diffuse interstellar medium through absorption measurements (Neufeld et al. 2010) and in emission in the Orion Bar (van der Tak et al. 2012). Neufeld et al. (2010) found a lower limit for the HF abundance of $6 \times 10^{-9}$ relative to hydrogen nuclei, providing support to the theoretical prediction that HF is the dominant fluorine reservoir among a wide variety of interstellar conditions. Unlike HF and $\mathrm{C}^{+}, \mathrm{CF}^{+}$rotational lines can be observed from the ground. However, up to now there has been only one detection of $\mathrm{CF}^{+}$ toward the Orion Bar (Neufeld et al. 2006).

In this Letter we report the detection of $\mathrm{CF}^{+}$at the $\mathrm{HCO}$ and $\mathrm{DCO}^{+}$peak emission, corresponding to the photo-dissociation region (PDR) and dense core environments in the Horsehead nebula, respectively (Pety et al. 2007; Gerin et al. 2009). We then infer the gas phase fluorine abundance.

\section{Observations and data reduction}

Figure 1 displays deep integrations of the $J=1-0$ and $J=$ 2-1 low-energy rotational lines of $\mathrm{CF}^{+}$with the IRAM-30m telescope centered at the PDR and the dense core, located at $(\delta \mathrm{RA}, \delta \mathrm{Dec})=\left(-5^{\prime \prime}, 0^{\prime \prime}\right)$ and $\left(20^{\prime \prime}, 22^{\prime \prime}\right)$ with respect to the projection center, with $49 \mathrm{kHz}$ spectral resolution at both frequencies. These observations were obtained as part of the Horsehead WHISPER project (Wideband High-resolution Iram-30m Surveys at two Positions with Emir Receivers). A presentation of the whole survey and the data reduction process will be given in Pety et al. (in prep.). 
Table 1. Observation parameters for the maps.

\begin{tabular}{|c|c|c|c|c|c|c|c|c|c|c|}
\hline Line & $\begin{array}{c}\text { Frequency } \\
\mathrm{GHz}\end{array}$ & $\overline{\text { Instrument }}$ & 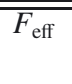 & 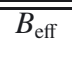 & $\begin{array}{l}\text { Beam } \\
\text { arcsec }\end{array}$ & $\overline{\mathrm{PA}}$ & $\begin{array}{c}\text { Int. time } \\
\mathrm{h}\end{array}$ & $\begin{array}{c}T_{\text {sys }} \\
\mathrm{K}\left(T_{\mathrm{A}}^{*}\right)\end{array}$ & $\begin{array}{c}\text { Noise } \\
\mathrm{K}\left(T_{\mathrm{mb}}\right)\end{array}$ & Obs. date \\
\hline $\mathrm{HCO} 1_{0,1} 3 / 2,2-0_{0,0} 1 / 2,1$ & 86.670760 & $\mathrm{PdBI} / \mathrm{C} \& \mathrm{D}$ & 0.95 & 0.78 & $6.7 \times 4.4$ & 16 & 6.5 & 150 & 0.09 & $2006-2007$ \\
\hline $\mathrm{CF}^{+} 1-0$ & 102.587533 & $30 \mathrm{~m} / \mathrm{EMIR}$ & 0.94 & 0.79 & 25.4 & 0 & 2.5 & 88 & $0.03^{b}$ & Jan. 2012 \\
\hline $\mathrm{CF}^{+} 2-1$ & 205.170520 & $30 \mathrm{~m} / \mathrm{EMIR}$ & 0.94 & 0.64 & 11.4 & 0 & 3.4 & 220 & $0.18^{c}$ & Jan. 2012 \\
\hline $\mathrm{DCO}^{+} 3-2$ & 216.112582 & $30 \mathrm{~m} / \mathrm{HERA}$ & 0.90 & 0.52 & 11.4 & 0 & 1.5 & 230 & 0.10 & Mar. 2006 \\
\hline
\end{tabular}

Notes. Their projection center is $\alpha_{2000}=05^{\mathrm{h}} 40^{\mathrm{m}} 54.27^{\mathrm{s}}, \delta_{2000}=-02^{\circ} 28^{\prime} 00^{\prime \prime} .{ }^{(a)}$ On-source integration time. The noise values are for a spectral resolution of ${ }^{(b)} 0.114 \mathrm{~km} \mathrm{~s}^{-1}$ and ${ }^{(c)} 0.057 \mathrm{~km} \mathrm{~s}^{-1}$.
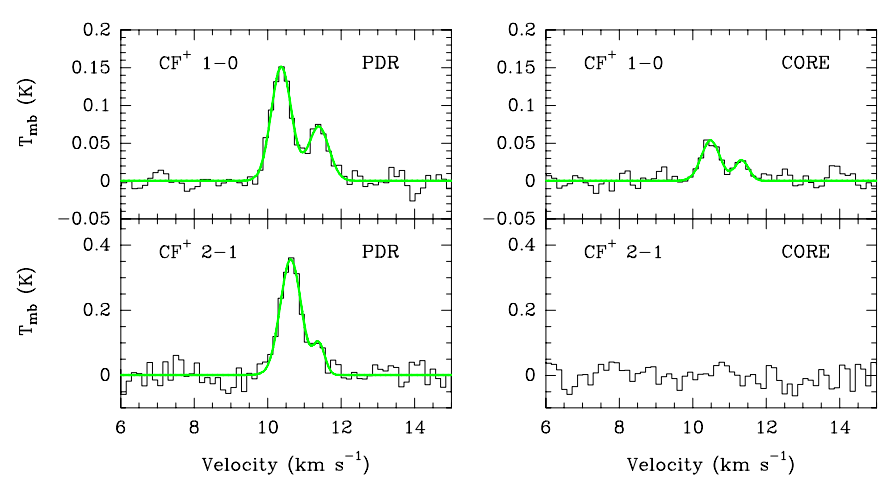

Fig. 1. Deep integrations toward the PDR and core positions. The green lines are double Gaussian fits.

The $\mathrm{CF}^{+} J=1-0$ and $J=2-1$ maps displayed in Fig. 2 were observed simultaneously during seven hours of good winter weather ( $2 \mathrm{~mm}$ of precipitable water vapor) using the EMIR sideband-separation receivers at the IRAM-30m. We used the position-switching, on-the-fly observing mode. The off-position offsets were $(\delta \mathrm{RA}, \delta \mathrm{Dec})=\left(-100^{\prime \prime}, 0^{\prime \prime}\right)$, i.e. into the HII region ionized by $\sigma$ Ori and free of molecular emission. We observed along and perpendicular to the direction of the exciting star in zigzags, covering an area of $100^{\prime \prime} \times 100^{\prime \prime}$. A description of the $\mathrm{HCO}$ and $\mathrm{DCO}^{+}$observations and data reductions, which are also displayed in Fig. 2, can be found in Gerin et al. (2009) and Pety et al. (2007). Table 1 summarizes the observation parameters for all these maps.

The maps were processed with the GILDAS ${ }^{1}$ softwares (Pety 2005). The IRAM-30m data were first calibrated to the $T_{\mathrm{A}}^{*}$ scale using the chopper-wheel method (Penzias \& Burrus 1973), and finally converted into main-beam temperatures $\left(T_{\mathrm{mb}}\right)$ using the forward and main-beam efficiencies $\left(F_{\text {eff }} \& B_{\text {eff }}\right)$ displayed in Table 1 . The resulting amplitude accuracy is $\sim 10 \%$. The resulting spectra were then baseline-corrected and gridded through convolution with a Gaussian to obtain the maps.

\section{Results}

\subsection{Line profiles}

Two velocity peaks for the $J=1-0$ line are clearly seen at both positions in Fig. 1. The second velocity peak is marginal for the $J=2-1$ line. Table 2 presents the results of dual Gaussian fits. The centroid velocity of each peak is significantly shifted between the two $\mathrm{CF}^{+}$transitions. We have carefully checked that neither peak is a residual line incompletely rejected from the image side band. This doubled-peak behavior is unexpected because all species without an hyperfine structure

\footnotetext{
1 See http://www.iram.fr/IRAMFR/GILDAS for more information about the GILDAS softwares.
}
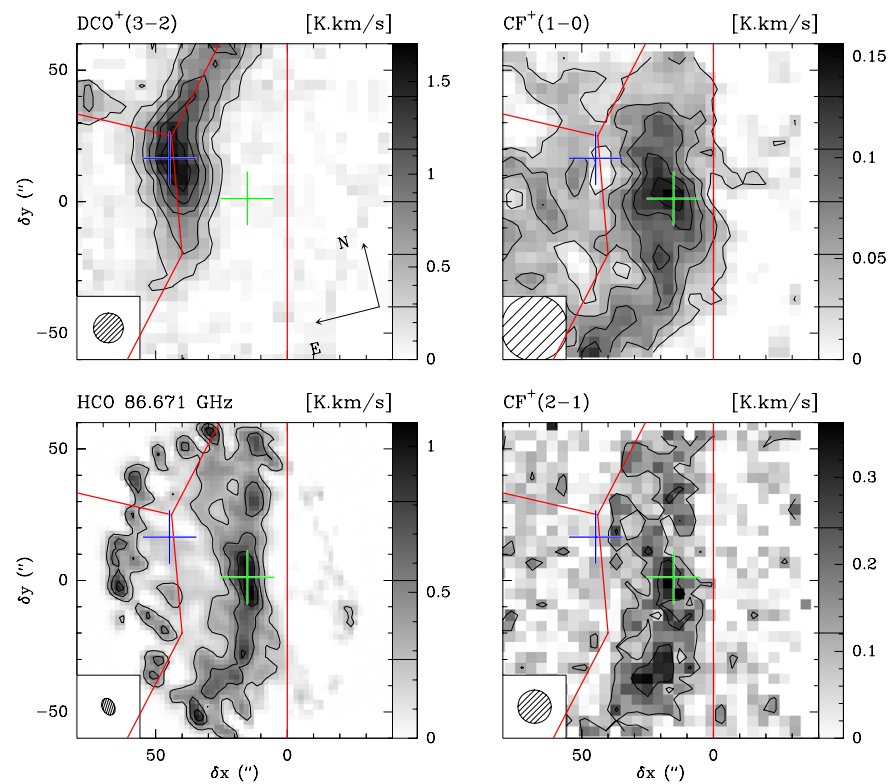

Fig. 2. Integrated intensity maps of the Horsehead edge. Maps were rotated by $14^{\circ}$ counter-clockwise around the projection center, located at $(\delta x, \delta y)=\left(20^{\prime \prime}, 0^{\prime \prime}\right)$, to bring the exciting star direction in the horizontal direction and the horizontal zero was set at the PDR edge, delineated by the red vertical line. The crosses show the positions of the PDR (green) and the dense core (blue). The spatial resolution is plotted in the bottom left corner. Values of the contour levels are shown in the color look-up table of each image (first contour at $2 \sigma$ and $2.5 \sigma$ for $\mathrm{CF}^{+} 1-0$ and $2-1$ respectively). The emission of all lines is integrated between 10.1 and $11.1 \mathrm{~km} \mathrm{~s}^{-1}$.

detected previously at millimeter wavelengths in the Horsehead present a simple velocity profile centered close to $10.7 \mathrm{~km} \mathrm{~s}^{-1}$. The only other species detected to date with a clear double peak emission profile is $\mathrm{C}^{+}$toward the illuminated edge of the cloud (Herschel/HIFI, Teyssier et al., in prep.).

The most obvious explanation would be that the higher velocity peak corresponds to another line from another species. However, there are no other lines in the public line catalogs (Pickett et al. 1998; Müller et al. 2001) near this frequency besides $\mathrm{CF}^{+}$. Another possible explanation would be that the two peaks correspond to different hyperfine components that are caused by the fluorine nuclei. To our knowledge, there are no hyperfine structure studies on $\mathrm{CF}^{+}$. However, because the molecule is isoelectronic with $\mathrm{CO}$, one can try to rely on ${ }^{13} \mathrm{CO}$ spectroscopy since the nuclear spin of ${ }^{13} \mathrm{C}$ is $1 / 2$, as for the fluorine nucleus. The magnetic dipolar coupling constant scales approximately with the rotational constant and the magnetic moment of the nucleus for ${ }^{1} \Sigma$ electronic ground states (Reid $\&$ Chu 1974). This allows us to derive a coupling constant of approximately $110 \mathrm{kHz}$, and an hyperfine splitting of $165 \mathrm{kHz}$ $(110 \mathrm{kHz})$ for the $J=1-0(J=2-1)$ transitions, well below 
V. Guzmán et al.: $\mathrm{CF}^{+}$as a tracer of $\mathrm{C}^{+}$and as a measure of the fluorine abundance

Table 2. Gaussian fit results.

\begin{tabular}{lcccc}
\hline \hline Line & $\begin{array}{c}\text { Line area } \\
\mathrm{K} \mathrm{km} \mathrm{s}^{-1}\end{array}$ & $\begin{array}{c}\text { Velocity } \\
\mathrm{km} \mathrm{s}^{-1}\end{array}$ & $\begin{array}{c}\text { Width } \\
\mathrm{km} \mathrm{s}^{-1}\end{array}$ & $\begin{array}{c}T_{\text {peak }} \\
\mathrm{K}\end{array}$ \\
\hline & $0.10 \pm 0.01$ & $10.36 \pm 0.02$ & $0.65 \pm 0.04$ & 0.15 \\
$\mathrm{CF}^{+} J=1-0$ & $0.05 \pm 0.01$ & $11.38 \pm 0.03$ & $0.66 \pm 0.09$ & 0.07 \\
& $0.25 \pm 0.02$ & $10.62 \pm 0.02$ & $0.67 \pm 0.06$ & 0.36 \\
$\mathrm{CF}^{+} J=2-1$ & $0.04 \pm 0.02$ & $11.39 \pm 0.06$ & $0.38 \pm 0.17$ & 0.09 \\
\hline \multicolumn{5}{c}{ CORE } \\
& $0.03 \pm 0.01$ & $10.47 \pm 0.04$ & $0.60 \pm 0.08$ & 0.05 \\
$\mathrm{CF}^{+} J=1-0$ & $0.01 \pm 0.01$ & $11.32 \pm 0.06$ & $0.45 \pm 0.20$ & 0.03 \\
$\mathrm{CF}^{+} J=2-1$ & $<0.05^{a}$ & $10.7^{a}$ & $0.5^{a}$ & $0.09^{a}$ \\
\hline
\end{tabular}

Notes. ${ }^{(a)}$ Upper limit for a fixed velocity, linewidth and a $T_{\text {peak }}=2 \sigma_{\text {rms }}$.

the observations. In addition, the respective intensities do not follow the theoretical predictions. Therefore, this possibility is unlikely. The profile is not caused by self-absorption because the $\mathrm{CF}^{+}$opacities are low $(\tau \lesssim 1)$. We therefore attribute the complex line profiles to kinematics in the $\mathrm{CF}^{+}$emitting layers.

\section{2. $\mathrm{CF}^{+}$spatial distribution}

Figure 2 presents the $\mathrm{CF}^{+}, \mathrm{HCO}$ and $\mathrm{DCO}^{+}$integrated line intensity maps. The $\mathrm{CF}^{+}$emission is concentrated toward the edge of the Horsehead, delineating the western edge of the $\mathrm{DCO}^{+}$emission. A more extended and fainter emission is detected in the $\mathrm{CF}^{+} J=1-0$ map but not in the $J=2-1$ map, which has a lower signal-to-noise ratio. The intensity peak of the $\mathrm{CF}^{+} J=1-0$ line coincides with the intensity peak of the $\mathrm{HCO}$ emission (shown by the green cross), which traces the far-UV illuminated matter (Gerin et al. 2009). The $J=2-1$ transition also peaks near the HCO emission peak.

We have checked that beam pick-up contamination from the PDR is negligible at the core position $(<7 \%)$, even with the large beam size $\left(\sim 25^{\prime \prime}\right)$ of the $30 \mathrm{~m}$ at $102 \mathrm{GHz}$. The emission then arises in the line of sight toward the core but not necessarily in the cold gas associated with the core. Indeed, we expect the $\mathrm{CF}^{+}$emission to arise in the outer layers of the nebula, delineating the edge as shown by the maps. This emission is likely to arise in the warmer and more diffuse material of the skin layers toward the core line of sight. Furthermore, there is a minimum of the emission in the $\mathrm{CF}^{+} 1-0$ map toward the dense core position. This confirms that the $\mathrm{CF}^{+}$emission is associated to the diffuse envelope. Molecular emission from the lower density cloud surface was already mentioned by Goicoechea et al. (2006) and Gerin et al. (2009) to explain the CS and HCO emission, respectively.

\subsection{Column densities and abundances}

The $\mathrm{CF}^{+}$column density was estimated assuming that the emission is optically thin and that the emission fills the $30 \mathrm{~m}$ beam. We inferred an excitation temperature of $10 \mathrm{~K}$, based on a rotational diagram built with the integrated line intensities of the two detected transitions. Assuming $T_{\mathrm{ex}}=10 \mathrm{~K}$ for all rotational levels, the beam averaged column density is $\simeq(1.5-2.0) \times 10^{12} \mathrm{~cm}^{-2}$ in the PDR. This value is similar to the column density found in the Orion Bar by Neufeld et al. (2006). In the next section, we will show that the $\mathrm{CF}^{+}$emission arises at the illuminated edge of the nebula. Goicoechea et al. (2009a) found that the [O I] $63 \mu \mathrm{m}$ fine structure line, which also arises at the edge of the nebula, was best reproduced with a gas density of $n_{\mathrm{H}} \simeq 10^{4} \mathrm{~cm}^{-3}$. Thus,

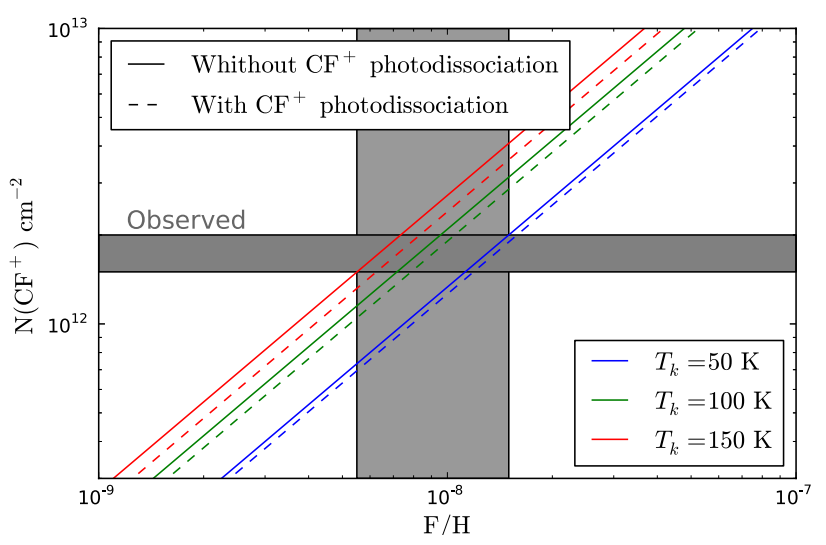

Fig. 3. Relation between the $\mathrm{CF}^{+}$column density and $\mathrm{F} / \mathrm{H}$.

assuming this density and a cloud depth $l \sim 0.1$ pc (Habart et al. 2005), the $\mathrm{CF}^{+}$column density translates into an abundance of $\simeq(4.9-6.5) \times 10^{-10}$ with respect to $\mathrm{H}$ nuclei. Taking the same excitation temperature of $10 \mathrm{~K}$, we computed a column density toward the core of $\simeq 4.4 \times 10^{11} \mathrm{~cm}^{-2}$. We consider this as an upper limit for the model in Sect. 3.4 because $\mathrm{CF}^{+}$is found in the surface layer, which is not taken into account by our unidimensional photochemical model.

\section{4. $\mathrm{CF}^{+}$chemistry}

$\mathrm{CF}^{+}$is formed through the following chemical path:

$$
\begin{gathered}
\mathrm{F}+\mathrm{H}_{2} \rightarrow \mathrm{HF}+\mathrm{H} \\
\mathrm{HF}+\mathrm{C}^{+} \stackrel{k_{1}}{\rightarrow} \mathrm{CF}^{+}+\mathrm{H}
\end{gathered}
$$

and destroyed by dissociative recombination with electrons (Neufeld et al. 2006) and by far-UV photons:

$$
\begin{aligned}
& \mathrm{CF}^{+}+\mathrm{e}^{-} \stackrel{k_{2}}{\longrightarrow} \mathrm{C}+\mathrm{F} \\
& \mathrm{CF}^{+}+h v \stackrel{k_{\mathrm{pd}}}{\longrightarrow} \mathrm{C}^{+}+\mathrm{F} .
\end{aligned}
$$

The reactions rates are $k_{1}=7.2 \times 10^{-9}(T / 300)^{-0.15} \mathrm{~cm}^{3} \mathrm{~s}^{-1}$ (Neufeld et al. 2005) and $k_{2}=5.3 \times 10^{-8}(T / 300)^{-0.8} \mathrm{~cm}^{3} \mathrm{~s}^{-1}$ (Novotny et al. 2005). The $\mathrm{CF}^{+}$photodissociation rate $k_{\mathrm{pd}}$ is not known. Nevertheless, assuming a rate of $10^{-9} \exp \left(-2.5 A_{\mathrm{V}}\right) \mathrm{s}^{-1}$, we estimate that this contribution is negligible compared to the dissociative recombination in low far-UV field PDRs like the Horsehead. However, it might not be negligible in regions with high-radiation fields $\left(\chi \simeq 10^{4}-10^{5}\right)$, like the Orion Bar. In the following we therefore assumed that $k_{\mathrm{pd}}=0$. Because 1) the fluorine chemistry is simple, 2) the electron abundance is given by the ionization of carbon $n(\mathrm{e}-) \sim n\left(\mathrm{C}^{+}\right)$and 3) $\mathrm{HF}$ is the major reservoir of fluorine $n(\mathrm{HF}) \sim n(\mathrm{~F})$, it can be shown that the $\mathrm{CF}^{+}$ column density is proportional to the fluorine gas phase abundance $([\mathrm{F}]=\mathrm{F} / \mathrm{H})$, i.e.

$N\left(\mathrm{CF}^{+}\right) \simeq \frac{k_{1}}{k_{2}}[\mathrm{~F}] n_{\mathrm{H}} l \quad\left[\mathrm{~cm}^{-2}\right]$

From our $\mathrm{CF}^{+}$observations we find $\mathrm{F} / \mathrm{H} \simeq(0.6-1.5) \times 10^{-8}$ in the Horsehead PDR (see Fig. 3), in good agreement with the solar value $\left(2.6 \times 10^{-8}\right.$; Asplund et al. 2009) and the one found in the diffuse atomic gas $\left(1.8 \times 10^{-8}\right.$; Snow et al. 2007). Sonnentrucker et al. (2010) also derived $\mathrm{F} / \mathrm{H} \simeq(0.5-0.8) \times 10^{-8}$ in diffuse molecular clouds detected in absorption with the Herschel/HIFI. 


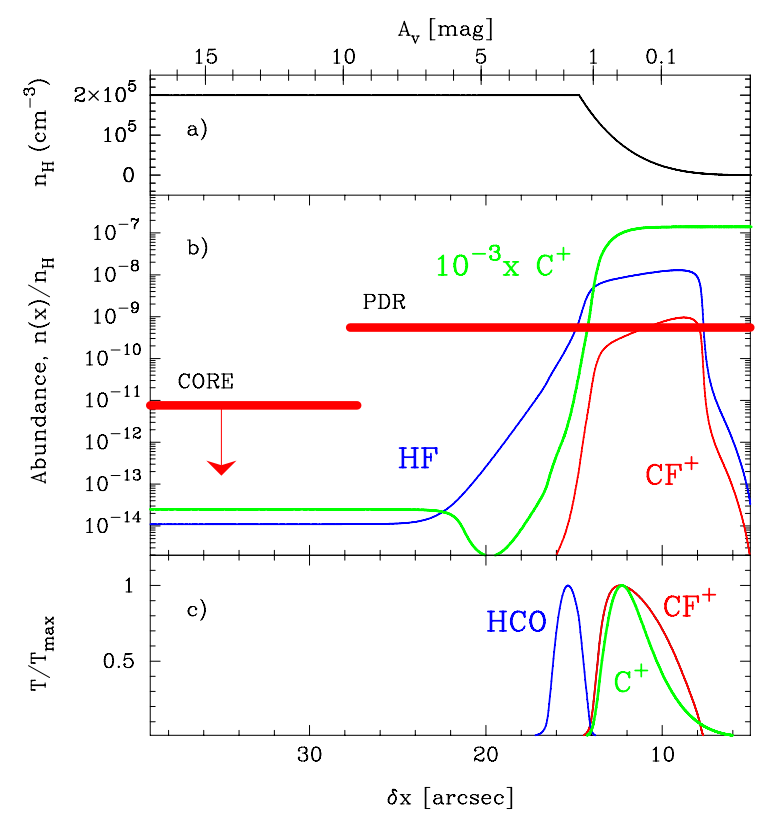

Fig. 4. Photochemical model of the Horsehead PDR. $A_{\mathrm{V}}$ increases from right to left; the PDR edge, delineated by the red vertical line in Fig. 2, corresponds to $A_{\mathrm{V}}=0$. a) Horsehead density profile $n_{\mathrm{H}}=n(\mathrm{H})+$ $2 n\left(\mathrm{H}_{2}\right)$. b) Predicted abundance of $\mathrm{CF}^{+}$in red, $\mathrm{HF}$ in blue and $\mathrm{C}^{+}$in green. The red horizontal bars show the measured $\mathrm{CF}^{+}$abundances, and their length represents the beam size. c) Predicted intensity profiles.

To understand the $\mathrm{CF}^{+}$abundance profile as a function of depth, we used a one-dimensional, steady-state photochemical model (Meudon PDR code, Le Bourlot et al. 2012; Le Petit et al. 2006). The version of the Meudon PDR model we used includes the Langmuir Hinshelwood and Eley-Rideal mechanisms to describe the formation of $\mathrm{H}_{2}$ on grain surfaces. The physical conditions in the Horsehead have already been constrained by our previous observational studies and we kept the same assumptions for the steep density gradient (displayed in the upper panel of Fig. 4), radiation field ( $\chi=60$ times the Draine 1978, mean interstellar radiation field), elemental gas-phase abundances (see Table 6 in Goicoechea et al. 2009b) and cosmic ray primary ionization rate $\left(\zeta=5 \times 10^{-17} \mathrm{~s}^{-1}\right.$ per $\mathrm{H}_{2}$ molecule). We used the Ohio State University (osu) pure gas-phase chemical network, and included fluorine adsorption and desorption onto grains.

The predicted $\mathrm{CF}^{+}, \mathrm{HF}$ and $\mathrm{C}^{+}$abundance profiles are shown in Fig. 4b. The $\mathrm{HF}$ and $\mathrm{CF}^{+}$abundances decrease rapidly for $A_{\mathrm{V}}>1$. The model agrees well with the observed $\mathrm{CF}^{+}$abundance in the PDR, shown by the horizontal bars. The model predicts that there is a significant overlap between $\mathrm{CF}^{+}$and $\mathrm{C}^{+}$. Moreover, the abundance ratio between these two species remains quite constant along the illuminated side of the cloud, i.e. $A_{\mathrm{V}}<4$, as shown in Fig. 4c. The $\mathrm{CF}^{+}$emission arises in the outermost layers of the cloud $\left(A_{\mathrm{V}} \sim 0.5\right)$, which are directly exposed to the far-UV field, where the gas is less dense. The predicted spatial distribution of the $\mathrm{CF}^{+}$emission is shown in Fig. 4d. We expect a narrow filament $\left(\sim 5^{\prime \prime}\right)$ shifted in the illuminated part of the PDR with respect to the $\mathrm{HCO}$ emission, which has already been shown to trace the far-UV illuminated molecular gas (Gerin et al. 2009).

\section{Conclusions}

We have detected the $J=1-0$ and $J=2-1$ rotational lines of $\mathrm{CF}^{+}$with high signal-to-noise ratio toward the PDR and core positions in the Horsehead. We have also mapped the region, and found that the emission arises mostly at the illuminated edge of the nebula (PDR), but it is also detected toward the dense core arising from its lower density skin. $\mathrm{CF}^{+}$ is unique because its column density is proportional to the elemental abundance of fluorine. In the Horsehead PDR we found $N \simeq(1.5-2.0) \times 10^{12} \mathrm{~cm}^{-2}$ and inferred $\mathrm{F} / \mathrm{H} \simeq(0.6-1.5) \times 10^{-8}$. Our model of the fluorine chemistry predicts that $\mathrm{CF}^{+}$accounts for $4-8 \%$ of all fluorine. $\mathrm{CF}^{+}$is found in the layers where $\mathrm{C}^{+}$is abundant because it is formed by reactions between $\mathrm{HF}$ and $\mathrm{C}^{+}$. In these regions the ionization fraction is high (see Goicoechea et al. 2009b) and $\mathrm{CF}^{+}$destruction is dominated by dissociative recombination with electrons. The $\mathrm{CF}^{+}$emission has two velocity components. The possibility that we are resolving the hyperfine structure is unlikely, but a corresponding theoretical or experimental study would allow us to derive the velocity structure unambiguously. Although the $\mathrm{CF}^{+}$line profile is not exactly the same as the $\mathrm{C}^{+}$line profile, they are the only species in the Horsehead with a double-peaked profile of kinematic origin measured to date. The complex line profile of both $\mathrm{CF}^{+}$and $\mathrm{C}^{+}$therefore confirms that they trace the gas directly exposed to the far-UV radiation, which shows a completely different kinematics than the following layers traced by other species, like $\mathrm{HCO}$. We therefore propose that $\mathrm{CF}^{+}$can be used as a proxy of the $\mathrm{C}^{+}$layers that can be observed from the ground. We will check this by comparing our findings with a Herschel/HIFI map of the $\mathrm{C}^{+}$emission in the Horsehead.

Acknowledgements. We thank the anonymous referee for useful comments that improved the Letter. V.G. acknowledges support from the Chilean Government through the Becas Chile scholarship program. This work was also funded by grant ANR-09-BLAN-0231-01 from the French Agence Nationale de la Recherche as part of the SCHISM project. J.R.G. thanks the Spanish MICINN for funding support through grants AYA2009-07304 and CSD2009-00038. J.R.G. is supported by a Ramón y Cajal research contract from the Spanish MICINN and co-financed by the European Social Fund.

\section{References}

Asplund, M., Grevesse, N., Sauval, A. J., \& Scott, P. 2009, ARA\&A, 47, 481 Draine, B. T. 1978, ApJS, 36, 595

Gerin, M., Goicoechea, J. R., Pety, J., \& Hily-Blant, P. 2009, A\&A, 494, 977

Goicoechea, J. R., Pety, J., Gerin, M., et al. 2006, A\&A, 456, 565

Goicoechea, J. R., Compiègne, M., \& Habart, E. 2009a, ApJ, 699, L165

Goicoechea, J. R., Pety, J., Gerin, M., Hily-Blant, P., \& Le Bourlot, J. 2009b, A\&A, 498, 771

Habart, E., Abergel, A., Walmsley, C. M., Teyssier, D., \& Pety, J. 2005, A\&A, 437, 177

Langer, W. D., Velusamy, T., Pineda, J. L., et al. 2010, A\&A, 521, L17

Le Bourlot, J., Le Petit, F., Pinto, C., Roueff, E., \& Roy, F. 2012, A\&A, 541, A76 Le Petit, F., Nehmé, C., Le Bourlot, J., \& Roueff, E. 2006, ApJS, 164, 506

Müller, H. S. P., Thorwirth, S., Roth, D. A., \& Winnewisser, G. 2001, A\&A, 370, L49

Neufeld, D. A., Wolfire, M. G., \& Schilke, P. 2005, ApJ, 628, 260

Neufeld, D. A., Schilke, P., Menten, K. M., et al. 2006, A\&A, 454, L37

Neufeld, D. A., Sonnentrucker, P., Phillips, T. G., et al. 2010, A\&A, 518, L108

Novotny, O., Mitchell, J. B. A., LeGarrec, J. L., et al. 2005, J. Phys. B At. Mol. Phys., 38, 1471

Penzias, A. A., \& Burrus, C. A. 1973, ARA\&A, 11, 51

Pety, J. 2005, in SF2A-2005: Semaine de l'Astrophysique Française, ed. F. Casoli, T. Contini, J. M. Hameury, \& L. Pagani, 721

Pety, J., Goicoechea, J. R., Hily-Blant, P., Gerin, M., \& Teyssier, D. 2007, A\&A, 464, L41

Pickett, H. M., Poynter, R. L., Cohen, E. A., et al. 1998, J. Quant. Spec. Radiat. Transf., 60, 883

Reid, R. V., \& Chu, A. H.-M. 1974, Phys. Rev. A, 9, 609

Snow, T. P., Destree, J. D., \& Jensen, A. G. 2007, ApJ, 655, 285

Sonnentrucker, P., Neufeld, D. A., Phillips, T. G., et al. 2010, A\&A, 521, L12

van der Tak, F. F. S., Ossenkopf, V., Nagy, Z., et al. 2012, A\&A, 537, L10 